\title{
Identifying Biomarkers for Evaluating Wound Extent and Age in the Contused Muscle of Rats Using Microarray Analysis: A Pilot Study
}

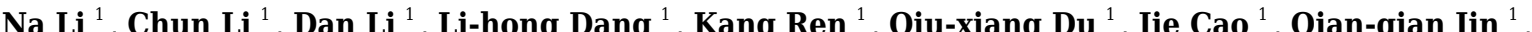
Ru-feng Bai ${ }^{\text {Corresp., } 2}$, Jun-hong Sun ${ }^{\text {Corresp. } 1}$

${ }^{1}$ School of Forensic Medicine, Shanxi Medical University, Jinzhong, China

2 Key Laboratory of Evidence Science, China University of Political Science and law, Beijing, China

Corresponding Authors: Ru-feng Bai, Jun-hong Sun

Email address: bairufeng@cupl.edu.cn, junhong.sun@sxmu.edu.cn

Wound age estimation is still one of the most important and significant challenges in forensic practice. The extent of wound damage greatly affects the accuracy and reliability of wound age estimation, so it is important to find effective biomarkers to help diagnose wound degree and wound age. In the present study, the gene expression profiles of both mild and severe injuries in 33 rats were assayed at $0,1,3,24,48$, and 168 hours using the Affymetrix microarray system to provide biomarkers for the evaluation of wound age and the extent of the wound. After obtaining thousands of differentially expressed genes, a principal component analysis, the least absolute shrinkage and selection operator, and a time-series analysis were used to select the most predictive prognostic genes. Finally, fifteen genes were screened for evaluating the extent of wound damage, and the top 60 genes were also screened for wound age estimation in mild and severe injury. Selected indicators showed good diagnostic performance for identifying the extent of the wound and wound age in a Fisher discriminant analysis. A function analysis showed that the candidate genes were mainly related to cell proliferation and the inflammatory response, primarily IL-17 and the Hematopoietic cell lineage signalling pathway. The results revealed that these genes play an essential role in wound-healing and yield helpful and valuable potential biomarkers for further targeted studies. 
1 Identifying Biomarkers for Evaluating Wound Extent

2 and Age in the Contused Muscle of Rats Using

3 Microarray Analysis: A Pilot Study

4

5

6

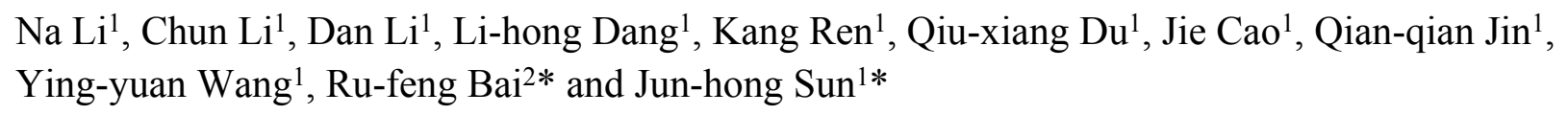

${ }^{1}$ School of Forensic Medicine, Shanxi Medical University, Jinzhong 030604, Shanxi, China;

${ }^{2}$ Key Laboratory of Evidence Science, China University of Political Science and law, Beijing, China; Collaborative Innovation Center of Judicial Civilization, Beijing, China

\section{Corresponding Author:}

Jun-hong Sun ${ }^{1}$

98 University Street, Yuci District, Jinzhong 030604, Shanxi, People's Republic of China.

Email address: junhong.sun@sxmu.edu.cn

Ru-feng Bai ${ }^{2}$

Chinese University of Political Science and Law, Beijing 100040, People's Republic of China Email address: bairufeng@,cupl.edu.cn

\section{Abstract}

Wound age estimation is still one of the most important and significant challenges in forensic practice. The extent of wound damage greatly affects the accuracy and reliability of wound age estimation, so it is important to find effective biomarkers to help diagnose wound degree and wound age. In the present study, the gene expression profiles of both mild and severe injuries in 33 rats were assayed at $0,1,3,24,48$, and 168 hours using the Affymetrix microarray system to provide biomarkers for the evaluation of wound age and the extent of the wound. After obtaining thousands of differentially expressed genes, a principal component analysis, the least absolute shrinkage and selection operator, and a time-series analysis were used to select the most predictive prognostic genes. Finally, fifteen genes were screened for evaluating the extent of wound damage, and the top 60 genes were also screened for wound age estimation in mild and severe injury. Selected indicators showed good diagnostic performance for identifying the extent of the wound and wound age in a Fisher discriminant analysis. A function analysis showed that the candidate genes were mainly related to cell proliferation and the inflammatory response, primarily IL-17 and the Hematopoietic cell lineage signalling pathway. The results revealed that 
35 these genes play an essential role in wound-healing and yield helpful and valuable potential 36 biomarkers for further targeted studies.

\section{Introduction}

38 In violent cases, an accurate estimation of wound age, which describes the time interval between 39 trauma infliction and death, is one of the most challenging points in forensic pathology (Casse et 40 al. 2016; Trautz et al. 2019; Wang et al. 2016). The precise estimation of wound age contributes 41 to rebuilding the scene of the case, demarcating the scope of suspects, determining the nature of 42 the cases, and finally facilitating the investigation process (Du et al. 2020; Sun et al. 2017). 43 However, the determination of wound age is influenced by many additional factors such as age, gender, concomitant diseases, therapy, and the extent of the wound damage making it even harder to ensure the accuracy and reliability of wound age prediction (Grellner \& Madea 2007; Li et al. 2018). Among these factors, the extent of a wound, which is highly variable among the muscles examined in forensic practice, may be the most important.

It is well known that wound age estimation mainly depends on the time-dependent expression patterns of the molecules involved in damage repair. Although skeletal muscle holds an innate capacity for self-repair after injury, fibrosis, or fatty muscle deposits with significant functional deficits, often occur in the case of severe injury. Fibrosis is related to both the different molecular responses after injury as well as the severity of the wound. Thus, the temporal expression pattern of molecules responding to injury varies with both the stage of the healing process and the severity of the injury. Most studies have concentrated solely on the timing of morphological and biochemical changes during wound healing (Hassan Gaballah et al. 2016; Ishida et al. 2015; Wang et al. 2015) and have ignored the effect of the level of wound damage. A careful evaluation of the extent of a wound is necessary to effectively determine wound age.

With the rapid development of life science and technology, particularly high-throughput technology, the measurement of damage parameters has improved, and more and more potential biomarkers for estimating the time of injury have been studied (Casse et al. 2016; Li et al. 2018). Microarray technology, which allows the simultaneous evaluation of the expression profiles of thousands of genes, has increasingly contributed to our understanding of many biological processes (Komamura et al. 2003; Li et al. 2017) and also shows great potential for identifying valuable biomarkers of the time-dependent expression for wound age estimation. The main 
66 problem with using microarray technology is that the number of genes greatly exceeds the

67 number of samples following a microarray experiment, leading to the main concern of how to 68 identify genes of interest from such a large gene set.

69 Fortunately, the least absolute shrinkage and selection operator (LASSO) regression model

70 was introduced with the potential to solve this problem. In a LASSO-penalized regression, as log

$71 \lambda$ (a tuning parameter) changes, the corresponding coefficients of certain genes are reduced to

72 zero, indicating that their effects on the model can be omitted because they are shrunk

73 parameters (Tibshirani 1997). LASSO regressions allow fewer hub genes to be obtained for

74 further analysis. Moreover, a time-series analysis, based on the multivariate empirical Bayes'

75 statistic (MBstatistic), is sufficient to find genes with large differences in their temporal patterns

76 between conditions and then rank them (Tai \& Speed 2009). We applied the LASSO method in

77 our study to evaluate the most informative prognostic mRNA biomarkers according to their

78

79

80

81

82

83

84

85

86

87

88

89

90

91

92

93

94

95 relative contribution to the prognosis of damage degree; we also used a time-series analysis to identify genes with large differences in temporal profiles for wound aging.

In a previous study, we established the muscle contusion models of rats caused by blunt force attack at different heights, and evaluated the gross and histological morphological changes over time as well as verified the repeatability of the model (Bai et al. 2017). Different numbers of inflammatory cells and different sizes of hemorrhage and edema were observed in mild and severe contusion muscles. In this study, we aimed to explore the molecular changes at the transcriptional level of mildly and severely contused muscle and identify valuable potential biomarkers for evaluating the extent of the wound as well as wound age. Because basic physiological responses are similar in both humans and animals, the predictive genes for wounds found in this study will provide valuable information for further forensic practice.

\section{Materials \& Methods}

\section{Animal model and tissue preparation}

All procedures were performed according to the "Guiding Principles in the Use and Care of Animals" (NIH Publication No. 85-23, Revised 1996) and were approved by the Institutional Animal Care and Use Committee of Shanxi Medical University of China [Batch number of rats:

4 SCXK (Jin) (2009-0001)]. Animals received humane care in conformity to the principles in the 5 Guide for the Care and Use of Laboratory Animals protocol, published by the Ministry of the 96 People's Republic of China (issued on June 4, 2004). The laboratory personnel were given 
97 permission to conduct this study by the Committee after they attended and completed training on 98 how to use experimental animals ethically. All male Sprague Dawley rats were purchased from 99 the Animal Center of Shanxi Medical University. This study was carried out in compliance with 100 the ARRIVE guidelines and evaluated and approved by the Institutional Animal Care and Use 101 Committee of Shanxi Medical University of China with approval number 2019s11002. 102 An animal model was established based on previous studies (Bai et al. 2017). In total, 33 103 male Sprague Dawley rats (aged 10-12 weeks), weighing 250-300g, were placed in a cage with rat chow and water, under a 12-hour light-dark cycle in a temperature-controlled room $\left(22-24^{\circ} \mathrm{C}\right)$ with a relative humidity of 40-60\%. No adverse events were observed. All rats were randomly divided into three groups:

(1) Control group $(n=3)$ : without any treatment before sampling.

(2) Mild contusion groups $(\mathrm{n}=15)$ : a $500 \mathrm{~g}$ counterpoise fell freely from a height of $15 \mathrm{~cm}$ down a clear Lucite guide tube onto the thigh muscles of the right posterior limb with the potential energy of the contusion totaling about $1.46 \mathrm{~J} / \mathrm{cm}^{2}$ (the formula: $\mathrm{Ep}=\mathrm{mgh}$ ).

(3) Severe contusion groups $(n=15)$ : a $500 \mathrm{~g}$ counterpoise fell freely from a height of $50 \mathrm{~cm}$, with the potential energy of contusion at about $2.58 \mathrm{~J} / \mathrm{cm}^{2}$ (the formula: $\mathrm{Ep}=\mathrm{mgh}$ ).

The animals in the mild and severely contused groups were further divided into five subgroups (1-, 3-, 24-, 48-, and 168-hour post-injury; $n=3$ /subgroup) for microarray analysis. Before muscle contusion, the rats were anesthetized with pentobarbital sodium to minimize the suffering of the rats during the experimental period. The rats in the contusion groups were sacrificed at the above timepoints with a lethal dose of pentobarbital (350 mg/kg body weight, intraperitoneal injection). Approximately $100 \mathrm{mg}$ of muscle was sampled from the wound site and equally divided into two parts from each rat. For the control rats, specimens were harvested from the same site after anesthetization with a lethal dose of pentobarbital. All the muscle samples from 33 rats were frozen immediately with liquid nitrogen for the microarray analysis.

A further 56 rats were randomly allocated to a control group $(n=8)$ and 4-, 8-, 12-, 16-, 20-, 24-, 28-, 32-, 36-, 40-, 44-, and 48-h (n=6/group) mild contusion groups for qPCR. Before contused, the right posterior limbs of the rats with pentobarbital were shaved by a depilatory agent (Nair; Carter Wallace, New York, NY, USA) and placed on an experimental table in a supine position. After damage, the rats were transferred into clean cages with food and water. 


\section{RNA extraction}

128 Total RNA was extracted as described in previous study (Du et al. 2020). Specifically, we 129 isolated the RNA from the skeletal muscle specimens (approximately $50 \mathrm{mg}$ each) using RNAiso 130 Plus 9108 (Takara Bio, Shiga, Japan), according to the manufacturer's protocol. The quantity 131 (ng/mL) and purity of RNA were accessed by a microplate reader (Infinite M200 Pro; TECAN, 132 Zurich, Switzerland), and the integrity of the RNA was further analyzed with an Agilent 2100 133 instrument (Agilent Technologies, Palo Alto, CA, USA) using an Agilent RNA 6000 Nano kit. 134 Only RNAs with OD260/OD280 ratios ranging from 1.8 to 2.2 and a RIN > 7.0 were selected for 135 the microarray and the RT-PCR (reverse transcription polymerase chain reaction).

\section{Microarray analysis}

137 Total RNA was used to synthesize sense-strand cDNA (sscDNA) using the Ambion WT 138 Expression Kit. Then, fragmentated and labeled sscDNA samples (using the Affymetrix ${ }^{\circledR}$ 139 GeneChip ${ }^{\circledR}$ WT Terminal Labeling Kit) were hybridized to Gene 1.1 ST Array Plates. Washed 140 and stained arrays were scanned using the GeneChip Scanner 3000 7G.

\section{Data processing}

142 Statistical significance of gene expression differences between the contusion and control groups 143 was analyzed using one-way ANOVA in the Affymetrix Transcriptome Analysis Console 144 software version 4.0.1. The expressions of mRNA with fold change $>2$ in mean expression 145 compared with the control group and a P-value of $<0.05$ and FDR (false discovery rate) $<0.05$ 146 were considered differentially expressed genes (DEGs).

147 A heatmap and an unsupervised hierarchical clustering analysis were performed to cluster 148 DEGs in the mild and severe groups, respectively, using the online software Morpheus 149 (https://software.broadinstitute.org/morpheus). Principal component analysis (PCA) was 150 employed to evaluate the importance of DEGs, distinguish the extent of contusions, and visualize the effect of the DEGs we selected in wound age estimation based on different wound degrees using SIMC A-P (ver. 14.1; Umetrics, Malmö, Sweden). T-distributed Stochastic Neighbor Embedding (t-SNE) was performed by R package "tsne" (version 0.15).

A least absolute shrinkage and selection operator (LASSO) regression model was then used to select key genes through a penalized likelihood approach (Tibshirani 1997). In the model, as $\log \lambda$ (a tuning parameter) changed, the regression coefficients penalize the size of the 
158 zero(Yan et al. 2020). Moreover, the "time course" R package was applied to find genes of 159 interest based on the multivariate empirical Bayes model. It was reported that this model was 160 superior to the traditional F-statistic for the analysis of time-series data (Tai \& Speed 2009). 161 Gene ontology (GO) annotation was conducted using the Gene Ontology Consortium 162 (http://geneontology.org) and the Kyoto encyclopedia of genes and genomes (KEGG) pathway 163 annotation was performed by clueGo plugin (https://apps.cytoscape.org/apps/cluego) (Bindea et 164 al. 2009). The protein-protein interaction (PPI) network was built by STRING 11.0 165 (https://string-db.org) and the networks were visualized and exported from Cytoscape 3.7.2 166 (Shannon et al. 2003). In addition, the top 10 hub genes in the PPI network were selected 167 according to the degree method using the plug-in CytoHubba in Cytoscape (Chin et al. 2014). A 168 Fisher discriminant analysis (FDA) was performed using SPSS software (SPSS version 24.0)

\section{Quantitative real-time PCR}

170 We prepared the reaction mixtures using the Premix Ex Taq TM kit (Takara Biotechnology Co.,

171 Ltd., Dalian, China) and then put them into the Bio-Rad CFX384 fluorescence Quantitative PCR 172 system (CFX384; Bio-Rad, Hercules, CA, USA) . Amplification was performed using a 2-step cycling program according to the following conditions: 40 cycles of 5 seconds at $95^{\circ} \mathrm{C}, 40$ seconds at $58-60^{\circ} \mathrm{C}$. Two reference (RPL13, ribosomal protein L13; RPL32, ribosomal protein L32) and two target genes were simultaneously assayed, as they were in previous studies (Dang et al. 2020; Du et al. 2020). Each reaction mixture consisted of Taq DNA polymerase (12.5 $\mu \mathrm{L})$, upstream and downstream primers and probes $(0.65 \mu \mathrm{L}$; eight primers and four probes $), 10 \%$ $(\mathrm{v} / \mathrm{v})$ DMSO $(2.0 \mu \mathrm{L})$, deionized water $(1.2 \mu \mathrm{L})$, and cDNA $(1.5 \mu \mathrm{L})$. The primers and probes that were used are listed in Table S1.

The flow diagrams, shown in Figure 1, summarize the experimental and data analysis procedures used in the present study. Transcriptional profiles in mildly and severely contused muscle After ruling out probes with low expressions, 16,005 probes were selected from 33 rats for the next analysis. There were 2,843 DGEs presented in the mild contusion groups and 2,298 DEGs in the severe contusion groups compared with the control group (Fig.2a, d). The number of DGEs found in mild injury was larger than that found in severe injury except at 168 hours. And at each time point, compared with the control group, the number of upregulated DGEs was more 
189 than down-regulated even with different degrees of injury (Fig.2b,2c). A PCA analysis of unique 190 genes showed that the mildly and severely contused groups were clearly separated from each 191 other, indicating that the severity of damage contributes the most to the variance in the data set

192 (Fig.2e).

\section{Hub genes to distinguish the extent of the injury}

194 The unique DEGs expressed between the mild and severe damage groups were then used to 195 assess the extent of wound damage. In a principal component analysis of unique genes, over $70 \%$ of the variance among the DEGs was retained in the first five principal components (PCs) (Fig.3a). The score of each principal component is displayed in Fig.3b-c and Fig.1S.a-c.

198

199

200

201

202

203

204

205

206

207

208

209

210

211

212

213

214

215

216

217

218

219

Although the variance of the first principal component (PC1) was the largest of the first five

principal components, PC2 captured most of the variance related to the genes expressed between the mild and severe groups (Fig.3a-c). As in Fig.3c, the mild and severe contusion groups were distributed in different areas on PC2, so the top 200 leading genes in PC2 were selected for further analysis.

A least absolute shrinkage and selection operator (LASSO) regression model was then used to select key genes (Tibshirani 1997). We conducted the LASSO algorithm with the R "glmnet" package (Friedman et al. 2010), and the optimal tuning parameter $(\lambda)$ was chosen to achieve the minimal partial likelihood deviance in the cross-validation plot (Fig.1S d-e). The fifteen genes with non-zero corresponding coefficients were entered into the multivariate model (Table S2). The PCA score plots showed that the control group and the mild and severe contusion groups were clearly separated from each other, indicating that the expression of 15 genes differed in mildly and severely contused muscle (Fig.3d). This conclusion was also supported by a random forest analysis (Fig.3e).

\section{The screening of candidate markers for injury time estimation in mild contusion}

\section{Candidate markers with time-series analysis}

An unsupervised hierarchical clustering analysis of 2843 DEGs in the mild contusion groups showed that the expression profiles of mild injury at the six observed time points could be segregated into three distinct clusters: the first cluster included contused muscle 24 and 48 hours after injury; the second cluster included contused muscle 0,1 , and 168 hours post-injury; and the third cluster included contused muscle 3 hours after injury (Fig.4a). PCA of all DEGs in mild injury also showed that the samples displayed a temporal separation in the PCA space (Fig.4b). 
220 One sample at $1 \mathrm{~h}$ that was clustered with the $3 \mathrm{~h}$ samples may be related to the fact that $1 \mathrm{~h}$ and $3 \mathrm{~h}$ 221 are very close and hierarchical clustering and PCA are unsupervised analyses with limited 222 discriminating ability. In addition, the fact that the samples of $1 \mathrm{~h}$ were grouped with $168 \mathrm{~h}$ may 223 be due to the similarity between the beginning and the end of the inflammation stage as both had 224 a few DEGs with similar expression profiles. Generally, the transcriptome profiles exhibited 225 time-dependent patterns that allowed us to identify useful markers of wound age.

226 For wound aging, the "time course" package in R language was used to obtain genes with 227 large differences in their temporal patterns over time after injury based on the empirical Bayes 228 model. Finally, the top 60 genes were chosen as candidate genes by their HotellingT2 value in 229 descending order (Table S3). The top 3 genes with different expression profiles in mild injury 230 were visualized and displayed in Fig.4c-e. A heatmap for the top 60 DEGs revealed that these 231 genes were expressed in four different temporal patterns, and the gene expression in these four 232 clusters were significantly increased over the period after injury (Fig.4f). Moreover, a t-SNE 233 analysis of these 60 DEGs showed different distributions for different contusion groups and that 234 they had the predictive potential for wound estimation in mild contusion groups (Fig.4g).

\section{Bioinformatics analysis of candidate markers}

236 To associate the changes of gene expressions with biological functions in mild injury, we 237 conducted a GO enrichment analysis of the top 60 genes to determine the enriched GO terms.

238 DEGs were categorized by their function into biological processes, cellular component, and 239 molecular function. $\mathrm{P}<0.05$ was used as a threshold to select significant $\mathrm{GO}$ categories in the 240 Gene Ontology Consortium and for KEGG pathway analysis in clueGo plugin (Fig.5a,5b). In 241 mild injury groups, we found that the top 3 categories of biological processes were related to 242 interspecies interaction between organisms, inflammatory response, and response to an external 243 stimulus. GO categories of cellular components were associated with myofibril, contractile fiber, 244 and sarcomere, and those related to molecular functions were relevant to cytokine activity, 245 signaling receptor activator activity, and receptor-ligand activity, suggesting the importance of 246 these genes during mild injury estimation. To identify the functional relationship among genes, a 247 PPI network of 60 genes was created and all genes had a STRING score of 0.7 or greater. We 248 used cytoHubba in Cytoscape to determine the hub genes, all of which play critical regulatory 249 roles in the PPI network. The top 15 genes with a high degree of connectivity were: Il1b, Il6, 
250 Cc12, I118, Cxc12, Timp1, Serpine1, Hmox1, Cxcr2, Cxc16, Mmp8, Cc17, Spp1, Ccr1 and Tnni1, 251 which were on the inside of the circle (Fig.5c).

252 The screening of candidate markers for severe injury time estimation

253 Candidate markers with time-series analysis

254 For expression profiling of the severely contused group, we used the same methods as in the 255 mildly contused groups. The PCA and unsupervised hierarchical clustering analysis of 2298

256 DEGs in the severe contusion groups revealed that samples from each time point were clustered 257 into a single group. Cluster analysis indicated two distinct transcriptome patterns of mild injury 258 in a time-dependent manner, which was supported by PCA results (Fig.6a, 6b). The empirical 259 Bayes model was also conducted using the 'time course' R package to rank genes based on their 260 expression over time. Lastly, the top 60 genes were acquired by their HotellingT2 values in 261 descending order for wound age estimation in severe contusion (Table S4). The top 3 genes with

262 different expression profiles in severe injury were visualized and displayed in Fig.6c-e. A

263 heatmap for the top 60 DEGs revealed that the majority of genes were highly expressed in the 264 late stage of injury (Fig.6f). Additionally, a t-SNE analysis of these 60 DEGs showed a 265 separation among the contused groups, revealing that their expression differed over time and 266 deserved to be explored further for wound age estimation (Fig.6g).

267 Bioinformatics analysis of candidate markers

268 In severely contused muscle, the top 3 categories of biological processes were related to defense 269 response, interspecies interaction between organisms, and response to external biotic stimulus.

270 Those of cellular components were associated with the external side of the plasma membrane, 271 and those of molecule functions were relevant to signaling receptor binding, cytokine activity, 272 and signaling receptor activator activity (Fig.7a). A KEGG analysis of the top 60 genes in the 273 clueGo plugin revealed that there were common pathways in both mild and severe injury

274 estimation such as the IL-17 signaling pathway and Hematopoietic cell lineage as well as unique 275 pathways in mild injury estimation including cytokine-cytokine receptor interaction and the TNF 276 signaling pathway (Fig.7b,7b). Most of these pathways were related to the process of injury and 277 repair, in which genes regulated the process of inflammation, for example, Cxc16, Ccr1, Csf3r, 278 Cxcr2, I118, Cc17, Cc12, I16, and Cxc12. Moreover, some pathways associated with bacterial 279 infection appeared such as Malaria and Salmonella infection, which may have close associations 280 with the inflammatory response. Using the STRING platform, the PPI network was built of these 
28160 genes in severe injury with a STRING score $\geqslant 0.7$. The top 15 hub genes determined by

282 cytoHubba were: Cd68, I16, Ptprc, Tyrobp, Cc12, Csf1r, C3ar1, Cybb, Fcgr1a, Cd53, Lgals3, 283 Timp1, Spp1, Tlr7, and Cd163 (Fig.7c).

\section{Validation of markers in mild injury by quantitative real-time PCR}

285 We validated the microarray data by examining the expression of Mt2a, Rrad, Gpnmb, and Timp1 (shared 286 genes of selected top60 genes of mild and serve injury) in the mild contusion groups using RT-qPCR

287 (Fig.8e-h). In previous studies, we verified the time-dependent expression of Mt2a mRNA in the contused 288 skeletal muscle of rats within 36 hours by RT-qPCR (Fan et al. 2017) (Fig.8e). Ling et al (Ling et al. 289 2016) found that Mt2a is closely associated with oxidative stress, mediated by subcellular pathways of 290 mitochondria, ER, lysosomal, and lipidosome, as well as the MAPKs (ERK, JNK, and p38) signal. In 291 present study, we continued to validate the expression of Rrad, Gpnmb, and Timp1(Fig.7f-h, Table S5) in 29256 rats. The expressions of Mt2a, Gpnmb, and Timp1 detected by RT-qPCR were elevated in a time 293 course manner, which was consistent with the results of the microarray (Fig.8a-d). Although the profiles 294 of Rrad were not identical to what we obtained from the microarray, mRNA of Rrad was upregulated 295 within $24 \mathrm{~h}$ compared with the control group and corresponded to the Affymetrix analyses. Collectively, 296 the consistency between the results of qPCR and the microarray analyses confirmed the reproducibility of 297 the microarray data.

\section{Performance of screened genes in Fisher discriminant analysis}

299 To validate the predictive and diagnostic performance of selected indicators, a Fisher 300 discriminant analysis (FDA) was employed. Based on the expression of fifteen hub genes, the 301 canonical functions can differentiate between the control, mild, and severe groups (Fig.9a). The 302 classification results showed 90.9\% accuracy for cross-validated grouped cases (seen in Table 303 S6), indicating that the molecules may respond differently during the repair process of mild and 304 severe injuries. For wound age estimation in mild injury, Lgmn, PVR, Hmox1, Egr1, Socs3, 305 Ankrd1, Cd8a, Pfkfb1, Msr1, Tnni1, and Myl3 of the top 60 candidate genes showed good 306 performance with only one wrongly classified sample of $168 \mathrm{~h} \mathrm{n}$ model of FDA (Fig.9b). In 307 severe injury, Apoe, Hmox1, Clec4a1, Egr1, Rrad, Il1rn, Akr1b8, C3ar1, Pltp, Ly49si1, and 308 Lilrb4 of the top 60 genes were included in the FDA model using the stepwise method and the 309 Wilks' lambda option. The results showed that all the samples were classified correctly (Fig.9c, 310 Table S7-8). Collectively, the fifteen hub genes and 120 candidate genes we provided showed 311 great potential for determining the extent of the wound and wound age and are valuable 312 candidates for further study. 


\section{Discussion}

314 Although a number of research projects have investigated how to determine the time of injuries,

315 significant barriers remain in the application of useful biomarkers to establish the chronology of 316 an injury in forensic practice. There are many factors that contribute to wound age estimation,

317 but one factor that plays as essential role in wound age estimates is the extent of the wound.

318 However, most studies focus on the changes at the morphological and molecular levels in bruises 319 over time without considering the impact of the force used to inflict the trauma. Barington $\mathrm{K}$ et

320 al. (Barington \& Jensen 2016) made an attempt to compare the changes of bruises inflicted with

321 a low, moderate, and high force, but they only explored bruises within $8 \mathrm{~h}$ at gross and

322 histological levels which is still far from practical applications.

323 In cases of violent injury, the severity of muscle bruising often varies among individuals 324 and among sites of damage within the same individual. Forensic pathologists mostly estimate 325 wound age based on the time-dependent changes of the molecules' response to an injury and the 326 morphological transformation caused by these molecules (Dang et al. 2020; Wang et al. 2016).

327 Many wound healing-related biomarkers have recently been explored for wound aging (Hassan 328 Gaballah et al. 2016; Ishida et al. 2018; Kubo et al. 2014); however, only a few markers have 329 sufficient diagnostic power for high-accuracy wound age estimation, which is partly because the 330 molecules respond differently at different levels of damage.

Therefore, it is important to identify useful biomarkers to evaluate the extent of a wound 332 and then to determine injury time. In this study, experimental animal models of muscle contusion 333 with different heights of blunt force were developed, and microarray technology was utilized to 334 identify a series of markers over time at the transcriptome level. Our study aimed to screen 335 useful markers to evaluate the extent of a wound, estimate damage time, and to be used for 336 further studies of wound age.

We first identified a total of 3,522 DEGs in mildly and severely contused muscle compared 338 to normal skeletal muscle in rats. According to PCA results, we found that unique genes can 339 better distinguish mild and severe injury than the genes that are shared between the two groups.

340 In order to discover indicators that contribute more to the discrimination of damage degrees, the 341 top 200 leading genes in PC2 of five principal components were chosen for subsequent analysis 342 (Fig.2c). In order to select the most powerful predictive prognostic genes for identifying the 343 extent of the wound damage, the least absolute shrinkage and selection operator (LASSO) 
344 regression model was used for variable shrinkage. Finally, 15 key genes were selected. The PCA 345 score plots showed that the control group and the mild and severe contusion groups were clearly 346 separate from each other, indicating that the expression of the 15 key genes differed in mildly

347 and severely contused muscle (Fig.3d-e). This conclusion was also supported by a random forest 348 analysis.

349 In addition, most of these 15 genes have been previously reported to play a vital role in cell 350 proliferation and the inflammatory response. Reducing APIP expression could help treat 351 systemic inflammatory response syndrome, a whole-body inflammatory state that can occur in 352 response to infection (Ko et al. 2012). Fam220a genes have been identified as novel target genes

353

354 355 356 357 358

359

360

361

362

363

364

365

366

367 368

369

370

371

372

373

374 for miR-489-3p and miR-92a-3p associated with renal injury and hypercholesterolemia (Wiese et al. 2019). Myadml2, which encodes myeloid-associated differentiation marker-like 2, may influence the ability of dairy cows to resist the inflammation response of mastitis (Chen et al. 2015). Trim72, with a RING finger domain, a B-box, two coiled-coil domains, and a SPRY domain, is specifically expressed in the plasma membrane of skeletal and cardiac muscle cells and is transcriptionally activated by the synergism of MyoD (or myogenin) and MEF2 (Cai et al. 2009; Jung \& Ko 2010; Lee et al. 2010). Ntn1, which encodes netrin 1, is a target of the Wnt/APC oncogenic pathways connected with the cell proliferation and inflammation response of hypoxia (Rosenberger et al. 2009; Zhou et al. 2015). GPS2, as a molecular guardian, is necessary for precise control of inflammatory responses involved in immunity and homeostasis (Cardamone et al. 2012; Venteclef et al. 2010). Yeats4, which encodes the YEATS domain containing 4, is closely related to cell proliferation in the cancer process (Ji et al. 2017; Jixiang et al. 2017; Pikor et al. 2013). Hivep2, which encodes human immunodeficiency virus type I enhancer-binding protein 2 , is associated with developmental delay, intellectual disability, and dysmorphic features (Steinfeld et al. 2016). These 15 genes have the potential to diagnose the extent of wounds and deserve to be explored further.

Having identified genes for evaluating the severity of a wound, we next used a time-series analysis based on the "time course" package in R language to find indicators for wound aging in the mild and severe contusion groups, respectively. As a result, the top 60 genes with large differences in temporal patterns among different time points post-injury were acquired for each group based on HotellingT2 values (Table S3-4). There were 30 genes that were shared by the mild and severe injury groups, and their expression profiles were strongly correlated with time in 
375 both contusion groups. This indicates that there were similar molecular and cellular responses

376 during the skeletal muscle repair process, although these responses were affected by the severity

377 of the injury. These shared genes were primarily involved in the inflammatory response and in

378 cytokine activity such as Ccr1, Il6, Ccl7, Pdpn, Spp1, Ccl2 and Timp1, which play vital roles in

379 the wound healing process. The expression of Mt2A, one of the 30 shared genes identified, was

380 verified in our previous study (Fan et al. 2017). In this study, the expression patterns of the

381 shared genes (Rrad, Gpnmb, and Timp1) were also examined using qPCR, which were generally

382 similar to microarray data; the results indicated that the data produced via the Affymetrix array

383 were reliable.

384 In the top 60 genes of mild injury, we found that the top 3 categories of biological processes

385 were related to interspecies interaction between organisms, and inflammatory response to an

386 external stimulus. GO categories of cellular components were associated with myofibril,

387 contractile fiber, and sarcomere, and those of molecule functions were relevant to cytokine

388 activity, signaling receptor activator activity, and receptor-ligand activity, suggesting the

389

390

391

392

393

394

395

396

397

398

399

400

401

402

403

404

405

importance of these genes during mild injury estimation. However, in the top 60 genes of severe injury, the top 3 categories of biological processes were related to defense response, interspecies interaction between organisms, and response to external biotic stimulus. Those of cellular components were associated with the external side of the plasma membrane, and those of molecule functions were relevant to signaling receptor binding, cytokine activity, and signaling receptor activator activity. There were also common pathways in mild and severe injury estimations such as the IL-17 signaling pathway and the Hematopoietic cell lineage, as well as unique pathways in mild injury estimation including cytokine-cytokine receptor interaction and the TNF signaling pathway (Fig.5c,7c). The genes identified in both mild and severe injury were correlated with cell proliferation and the process of inflammation, indicating that time-dependent genes play an important role in repair after injury.

This study identified different criteria and methods of biomarker identification for evaluating the extent of wounds and wound age. In order to identify genes to distinguish the extent of the injury, the unique DEGs, which were only significantly changed in the mild or severe damage groups compared with the control groups, were selected to identify the differences between mild and severe contusions. The expression profiles of the fifteen genes selected for evaluating the extent of the damage differed more in mildly and severely contused

Peer) reviewing PDF | (2021:08:64995:1:1:NEW 30 Nov 2021) 
406 muscle than they did when evaluating wound age. Conversely, for wound age estimation, our

407 goal was to find genes expressed over time that contained different information at different time 408 points in mild or severe injury. Therefore, the top 60 genes with a large difference in their 409 temporal patterns were screened for wound age estimation in the mild and severe contusion 410 groups, respectively. All these efforts were made to help improve diagnostic accuracy and 411 narrow the margin of error for wound age estimation based on distinguishing the degree of 412 damage.

413 In this study, microarray analyses were performed to characterize the transcriptional 414 expression changes of mildly and severely contused muscle collected at different time points 415 postinjury. After obtaining thousands of DEGs, PCA, LASSO regression, and time-series 416 analyses were used to select the most powerful predictive genes. Finally, fifteen genes for 417 evaluating the extent of wound damage and the top 60 genes for wound age estimation in mild 418 and severe injury were respectively screened. A functional enrichment analysis revealed that 419 these selected genes are actively involved in the muscle repair process and deserve to be 420 addressed and explored further in relation to wound age estimation.

\section{Conclusions}

422 For an accurate assessment of wound age, while considering the effect of wound severity, 423 we carried out a transcriptome-based microarray assay to obtain the expression profiles of wound 424 healing genes following mild and severe muscle contusions. Using statistical and bioinformatics 425 analyses, diagnostic genes were identified for evaluating the extent of wounds and wound age in 426 future studies. However, there are limitations to this study. The sample size for the microarray 427 analysis was small; further research with larger samples is necessary to validate more biomarkers 428 in contused muscle with different degrees of damage.

\section{Data Availability}

430 The microarray data in this study have been uploaded to GEO (accession number: GSE162565). 


\section{References}

434 Bai RF, Lü XJ, E XF, Yu TS, Liu R, and Zhang HD. 2017. Comparison of the Skin and Skeletal

435

436

437

438

439

440

441

442

443

444

445

446

447

448

449

450

451

452

453

454

455

456

457

458

459

460

461

462

463

464

465

466

467

468

469

470

471

472 Muscle Contusion in Rats Induced by Blunt Force with Different Heights. Journal of forensic medicine 33:1-5. 10.3969/j.issn.1004-5619.2017.01.001

Barington K, and Jensen HE. 2016. The impact of force on the timing of bruises evaluated in a porcine model. Journal of forensic and legal medicine 40:61-66. 10.1016/j.jflm.2016.03.005

Bindea G, Mlecnik B, Hackl H, Charoentong P, Tosolini M, Kirilovsky A, Fridman WH, Pagès F, Trajanoski Z, and Galon J. 2009. ClueGO: a Cytoscape plug-in to decipher functionally grouped gene ontology and pathway annotation networks. Bioinformatics 25:1091-1093. 10.1093/bioinformatics/btp101

Cai C, Masumiya H, Weisleder N, Matsuda N, Nishi M, Hwang M, Ko JK, Lin P, Thornton A, Zhao X, Pan Z, Komazaki S, Brotto M, Takeshima H, and Ma J. 2009. MG53 nucleates assembly of cell membrane repair machinery. Nature cell biology11:56-64. 10.1038/ncb1812

Cardamone MD, Krones A, Tanasa B, Taylor H, Ricci L, Ohgi KA, Glass CK, Rosenfeld MG, and Perissi V. 2012. A protective strategy against hyperinflammatory responses requiring the nontranscriptional actions of GPS2. Molecular cell 46:91-104. 10.1016/j.molcel.2012.01.025

Casse JM, Martrille L, Vignaud JM, and Gauchotte G. 2016. Skin wounds vitality markers in forensic pathology: An updated review. Medicine, science, and the law 56:128-137. $10.1177 / 0025802415590175$

Chen X, Cheng Z, Zhang S, Werling D, and Wathes D. 2015. Combining Genome Wide Association Studies and Differential Gene Expression Data Analyses Identifies Candidate Genes Affecting Mastitis Caused by Two Different Pathogens in the Dairy Cow. Open Journal of Animal Sciences 5:358-393. doi: 10.4236/ojas.2015.54040.

Chin $\mathrm{CH}$, Chen SH, Wu HH, Ho CW, Ko MT, and Lin CY. 2014. cytoHubba: identifying hub objects and sub-networks from complex interactome. BMC systems biology 8 Suppl 4:S11. 10.1186/1752-0509-8-s4-s11

Dang LH, Feng N, An GS, Cao J, Du QX, Jin QQ, Huang P, and Sun JH. 2020. Novel insights into wound age estimation: combined with "up, no change, or down" system and cosine similarity in python environment. International journal of legal medicine 134:2177-2186. $10.1007 / \mathrm{s} 00414-020-02411-\mathrm{z}$

Du QX, Li N, Dang LH, Dong TN, Lu HL, Shi FX, Jin QQ, Jie C, and Sun JH. 2020. Temporal expression of wound healing-related genes inform wound age estimation in rats after a skeletal muscle contusion: a multivariate statistical model analysis. International journal of legal medicine 134:273-282. 10.1007/s00414-018-01990-2

Fan HL, Liu SF, Sun JH, and Wang YY. 2017. Time-dependent Expression of MT1A mRNA and MT2A mRNA in the Contused Skeletal Muscle of Rats. Journal of forensic medicine 33:610. 10.3969/j.issn.1004-5619.2017.01.002

Friedman J, Hastie T, and Tibshirani R. 2010. Regularization Paths for Generalized Linear Models via Coordinate Descent. Journal of statistical software 33:1-22.

Grellner W, and Madea B. 2007. Demands on scientific studies: Vitality of wounds and wound 
473

474

475

476

477

478

479

480

481

482

483

484

485

486

487

488

489

490

491

492

493

494

495

496

497

498

499

500

501

502

503

504

505

506

507

508

509

510

511

512

age estimation. Forensic Science International 165:150-154. 10.1016/j.forsciint.2006.05.029 Hassan Gaballah M, Fukuta M, Maeno Y, Seko-Nakamura Y, Monma-Ohtaki J, Shibata Y, Kato H, Aoki Y, and Takamiya M. 2016. Simultaneous time course analysis of multiple markers based on DNA microarray in incised wound in skeletal muscle for wound aging. Forensic Science International 266:357-368. 10.1016/j.forsciint.2016.06.027

Ishida Y, Kuninaka Y, Nosaka M, Kimura A, Kawaguchi T, Hama M, Sakamoto S, Shinozaki K, Eisenmenger W, and Kondo T. 2015. Immunohistochemical analysis on MMP-2 and MMP9 for wound age determination. International journal of legal medicine 129:1043-1048. 10.1007/s00414-015-1167-5

Ishida Y, Kuninaka Y, Nosaka M, Shimada E, Hata S, Yamamoto H, Hashizume Y, Kimura A, Furukawa F, and Kondo T. 2018. Forensic application of epidermal AQP3 expression to determination of wound vitality in human compressed neck skin. International journal of legal medicine 132:1375-1380. 10.1007/s00414-018-1780-1

Ji S, Zhang Y, and Yang B. 2017. YEATS Domain Containing 4 Promotes Gastric Cancer Cell Proliferation and Mediates Tumor Progression via Activating the Wnt/ $\beta$-Catenin Signaling Pathway. Oncology research 25:1633-1641. 10.3727/096504017x14878528144150

Jixiang C, Shengchun D, Jianguo Q, Zhengfa M, Xin F, Xuqing W, Jianxin Z, and Lei C. 2017. YEATS4 promotes the tumorigenesis of pancreatic cancer by activating beta-catenin/TCF signaling. Oncotarget 8:25200-25210. 10.18632/oncotarget.15633

Jung SY, and Ko YG. 2010. TRIM72, a novel negative feedback regulator of myogenesis, is transcriptionally activated by the synergism of MyoD (or myogenin) and MEF2. Biochemical and biophysical research communications 396:238-245. 10.1016/j.bbrc.2010.04.072

Ko DC, Gamazon ER, Shukla KP, Pfuetzner RA, Whittington D, Holden TD, Brittnacher MJ, Fong C, Radey M, Ogohara C, Stark AL, Akey JM, Dolan ME, Wurfel MM, and Miller SI. 2012. Functional genetic screen of human diversity reveals that a methionine salvage enzyme regulates inflammatory cell death. Proceedings of the National Academy of Sciences of the United States of America 109:E2343-2352. 10.1073/pnas.1206701109

Komamura K, Shirotani-Ikejima H, Tatsumi R, Tsujita-Kuroda Y, Kitakaze M, Miyatake K, Sunagawa K, and Miyata T. 2003. Differential gene expression in the rat skeletal and heart muscle in glucocorticoid-induced myopathy: analysis by microarray. Cardiovascular drugs and therapy 17:303-310. 10.1023/a:1027352703783

Kubo H, Hayashi T, Ago K, Ago M, Kanekura T, and Ogata M. 2014. Temporal expression of wound healing-related genes in skin burn injury. Legal medicine (Tokyo) 16:8-13. 10.1016/j.legalmed.2013.10.002

Lee CS, Yi JS, Jung SY, Kim BW, Lee NR, Choo HJ, Jang SY, Han J, Chi SG, Park M, Lee JH, and Ko YG. 2010. TRIM72 negatively regulates myogenesis via targeting insulin receptor substrate-1. Cell death and differentiation 17:1254-1265. 10.1038/cdd.2010.1

Li N, Du Q, Bai R, and Sun J. 2018. Vitality and wound-age estimation in forensic pathology: review and future prospects. Forensic Sciences Research 5:15-24. $10.1080 / 20961790.2018 .1445441$ 
513 Li Z, Pan J, Ma J, Zhang Z, and Bai Y. 2017. Microarray gene expression of periosteum in

514

515

516

517

518

519

520

521

522

523

524

525

526

527

528

529

530

531

532

533

534

535

536

537

538

539

540

541

542

543

544

545

546

547

548

549

550

551

552 spontaneous bone regeneration of mandibular segmental defects. Scientific reports 7:13535. 10.1038/s41598-017-13586-8

Ling XB, Wei HW, Wang J, Kong YQ, Wu YY, Guo JL, Li TF, and Li JK. 2016. Mammalian Metallothionein-2A and Oxidative Stress. International journal of molecular sciences 17. 10.3390/ijms 17091483

Pikor LA, Lockwood WW, Thu KL, Vucic EA, Chari R, Gazdar AF, Lam S, and Lam WL. 2013. YEATS4 is a novel oncogene amplified in non-small cell lung cancer that regulates the p53 pathway. Cancer research 73:7301-7312. 10.1158/0008-5472.can-13-1897

Rosenberger P, Schwab JM, Mirakaj V, Masekowsky E, Mager A, Morote-Garcia JC, Unertl K, and Eltzschig HK. 2009. Hypoxia-inducible factor-dependent induction of netrin-1 dampens inflammation caused by hypoxia. Nature immunology 10:195-202. 10.1038/ni.1683

Shannon P, Markiel A, Ozier O, Baliga NS, Wang JT, Ramage D, Amin N, Schwikowski B, and Ideker T. 2003. Cytoscape: a software environment for integrated models of biomolecular interaction networks. Genome research 13:2498-2504. 10.1101/gr.1239303

Steinfeld H, Cho MT, Retterer K, Person R, Schaefer GB, Danylchuk N, Malik S, Wechsler SB, Wheeler PG, van Gassen KL, Terhal PA, Verhoeven VJ, van Slegtenhorst MA, Monaghan KG, Henderson LB, and Chung WK. 2016. Mutations in HIVEP2 are associated with developmental delay, intellectual disability, and dysmorphic features. Neurogenetics 17:159164. 10.1007/s10048-016-0479-Z

Sun JH, Zhu XY, Dong TN, Zhang XH, Liu QQ, Li SQ, and Du QX. 2017. An "up, no change, or down" system: Time-dependent expression of mRNAs in contused skeletal muscle of rats used for wound age estimation. Forensic Science International 272:104-110. 10.1016/j.forsciint.2017.01.012

Tai YC, and Speed TP. 2009. On gene ranking using replicated microarray time course data. Biometrics 65:40-51. 10.1111/j.1541-0420.2008.01057.x

Tibshirani R. 1997. The lasso method for variable selection in the Cox model. Statistics in medicine 16:385-395. 10.1002/(sici)1097-0258(19970228)16:4<385::aid-sim380>3.0.co;2-3

Trautz F, Franke H, Bohnert S, Hammer N, Müller W, Stassart R, Tse R, Zwirner J, Dreßler J, and Ondruschka B. 2019. Survival-time dependent increase in neuronal IL-6 and astroglial GFAP expression in fatally injured human brain tissue. Scientific reports 9:11771. 10.1038/s41598019-48145-w

Venteclef N, Jakobsson T, Ehrlund A, Damdimopoulos A, Mikkonen L, Ellis E, Nilsson LM, Parini P, Jänne OA, Gustafsson JA, Steffensen KR, and Treuter E. 2010. GPS2-dependent corepressor/SUMO pathways govern anti-inflammatory actions of LRH-1 and LXRbeta in the hepatic acute phase response. Genes \& development 24:381-395. 10.1101/gad.545110

Wang L-L, Zhao R, Liu C-S, Liu M, Li S-S, Li J-Y, Jiang S-K, Zhang M, Tian Z-L, Wang M, Zhang M-Z, and Guan D-W. 2016. A fundamental study on the dynamics of multiple biomarkers in mouse excisional wounds for wound age estimation. Journal of forensic and legal medicine 39:138-146. 10.1016/j.jflm.2016.01.027 
553 Wang Y, Yamamoto Y, Kuninaka Y, Kondo T, and Furukawa F. 2015. Forensic Potential of 554 MMPs and CC Chemokines for Wound Age Determination. Journal of forensic sciences $555 \quad 60: 1511-1515.10 .1111 / 1556-4029.12831$

556 Wiese CB, Zhong J, Xu ZQ, Zhang Y, Ramirez Solano MA, Zhu W, Linton MF, Sheng Q, Kon

$557 \quad \mathrm{~V}$, and Vickers KC. 2019. Dual inhibition of endothelial miR-92a-3p and miR-489-3p reduces

558 renal injury-associated atherosclerosis. Atherosclerosis 282:121-131.

$559 \quad$ 10.1016/j.atherosclerosis.2019.01.023

560 Yan J, Wu L, Jia C, Yu S, Lu Z, Sun Y, and Chen J. 2020. Development of a four-gene prognostic 561 model for pancreatic cancer based on transcriptome dysregulation. Aging (Albany NY) $562 \quad 12: 3747-3770.10 .18632$ /aging.102844

563 Zhou Z, Xie J, Cai Y, Yang S, Chen Y, and Wu H. 2015. The significance of NTR1 expression 564 and its correlation with $\beta$-catenin and EGFR in gastric cancer. Diagnostic pathology 10:128. $565 \quad 10.1186 / \mathrm{s} 13000-015-0356-3$

566 
Figure 1

Fig.1. Flow diagram of the experimental design and data analysis used in the present study.

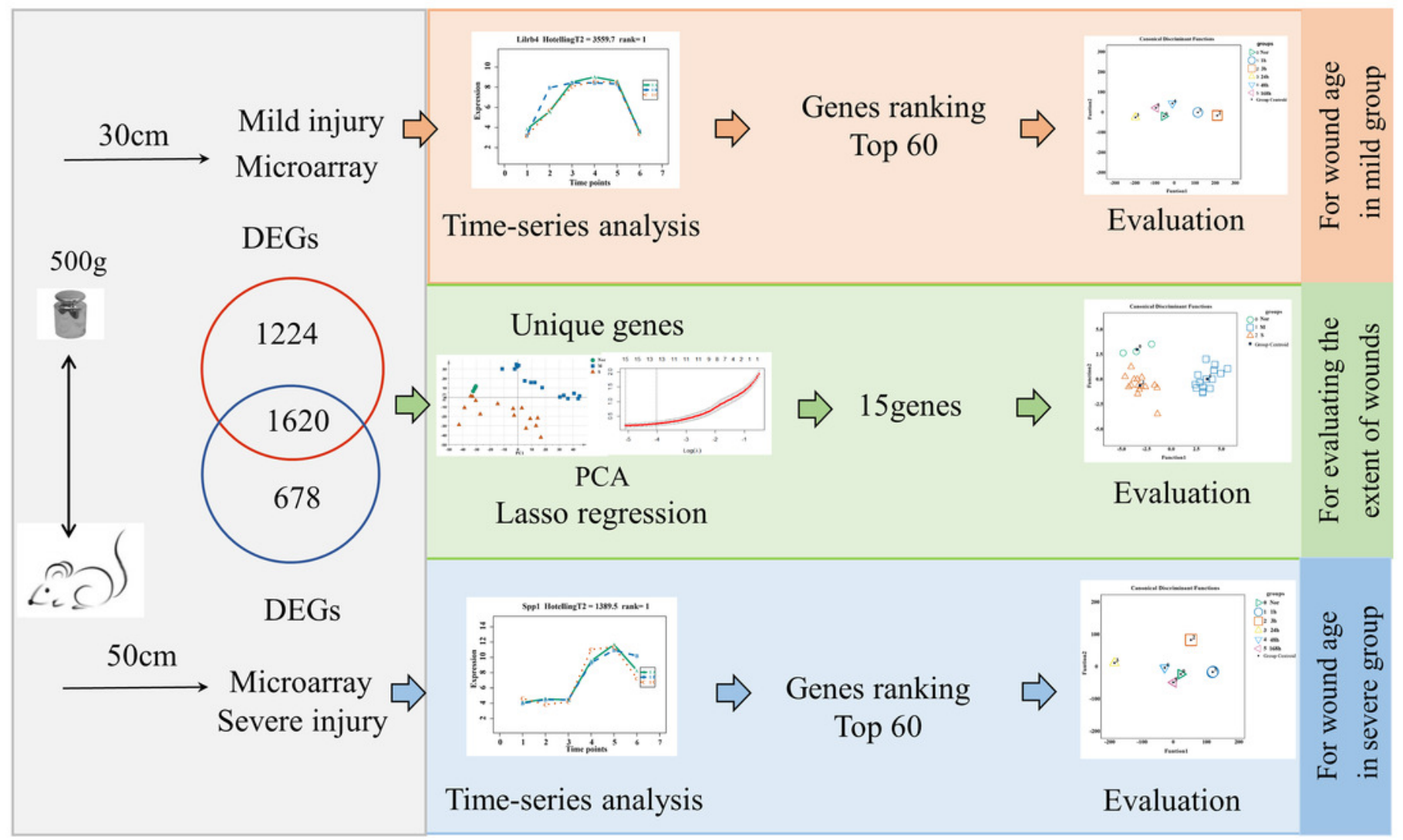


Figure 2

Fig.2. Dynamic transcriptome changes in mildly and severely contused muscle.

(a) The number of DEGs with $\mathrm{P}<0.05$ and 2 -fold change at each time point in the mild and severe contusion groups. (b) The number of upregulated and downregulated DEGs in the mild injury groups. (c) The number of upregulated and downregulated DEGs in the severe injury groups. (d) Venn diagram showing that there were 1,620 common genes shared and 1,903 unique genes between the mild and severe contusion groups. (e) The PCA result of unique genes between the mild and severe contusion groups; samples were colored according to the degree of damage. "Nor" means normal groups (in bluish green); "M" means mild injury groups (in blue); "S" means severe injury groups (in vermilion).

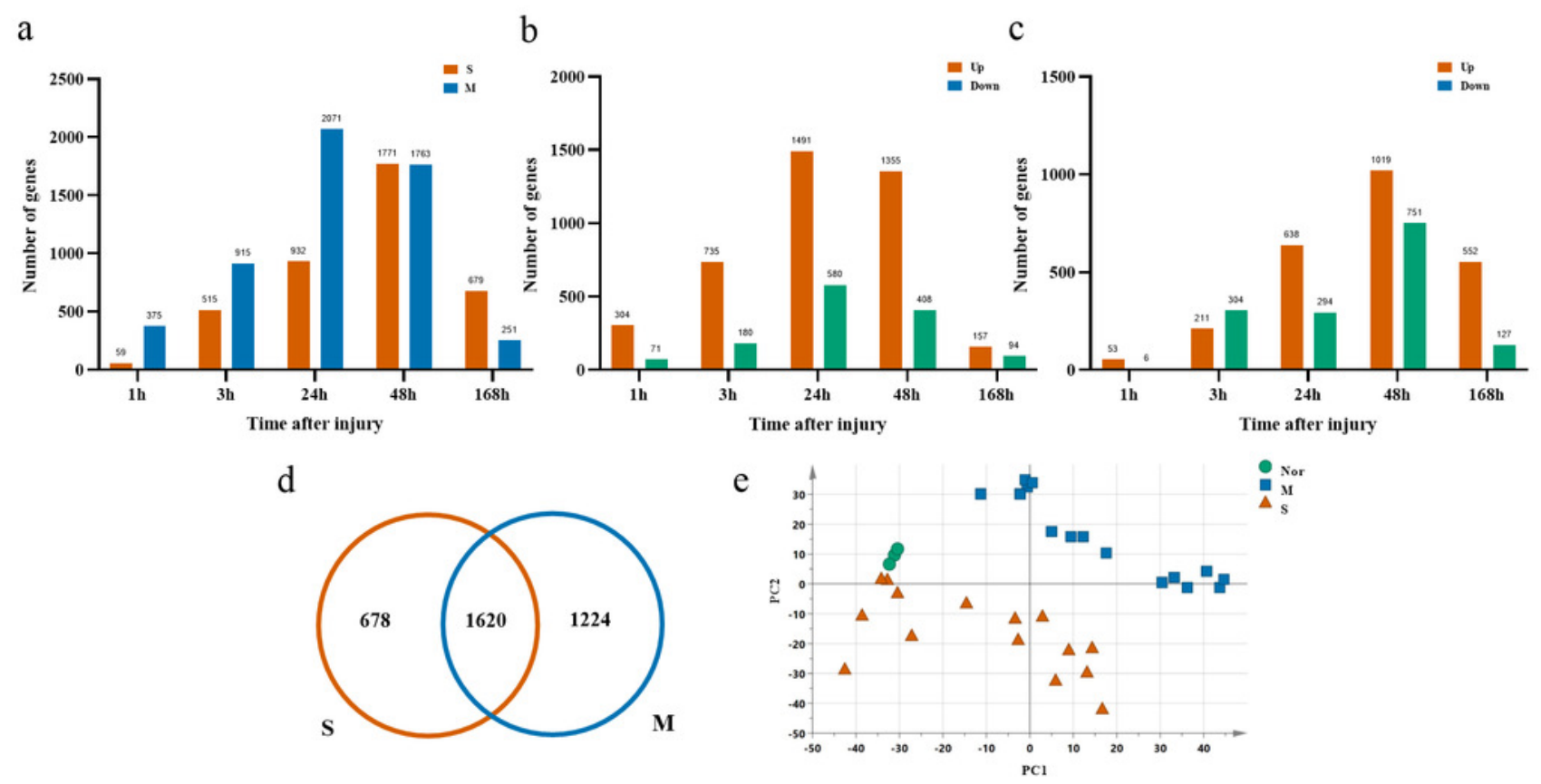




\section{Figure 3}

Fig.3. Identification of genes that drive the differences in gene expression between mild and severe injury.

(a) Percentage of variance explained by each principal component. (b-c) The score plots of principal component (PC)1 and PC2. (d-e) PCA (d) and Random Forest (e) of the 15 prognostic DEGs showed that the control group and the mild and severe contusion groups were clearly separated from each other. "Nor" means normal groups (in bluish green); "M" means mild injury groups (in blue); "S" means severe injury groups (in vermilion) .

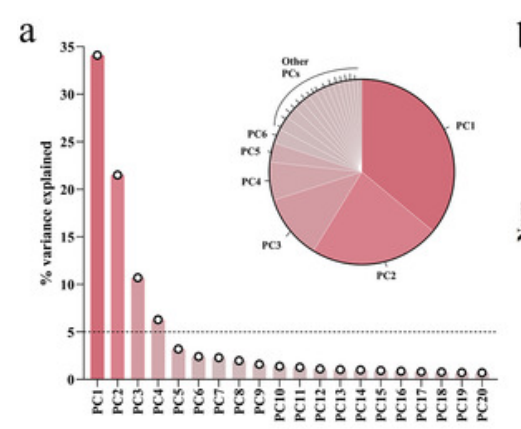$$
\text { d }
$$

$\mathrm{b}$

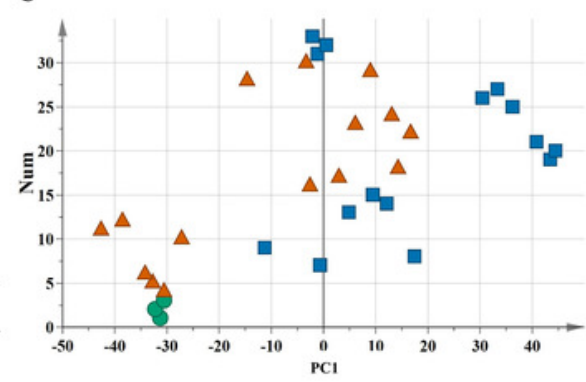

e

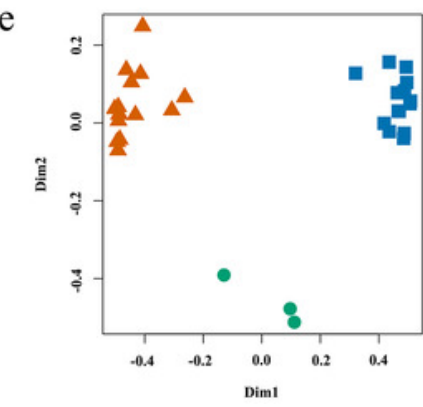

:
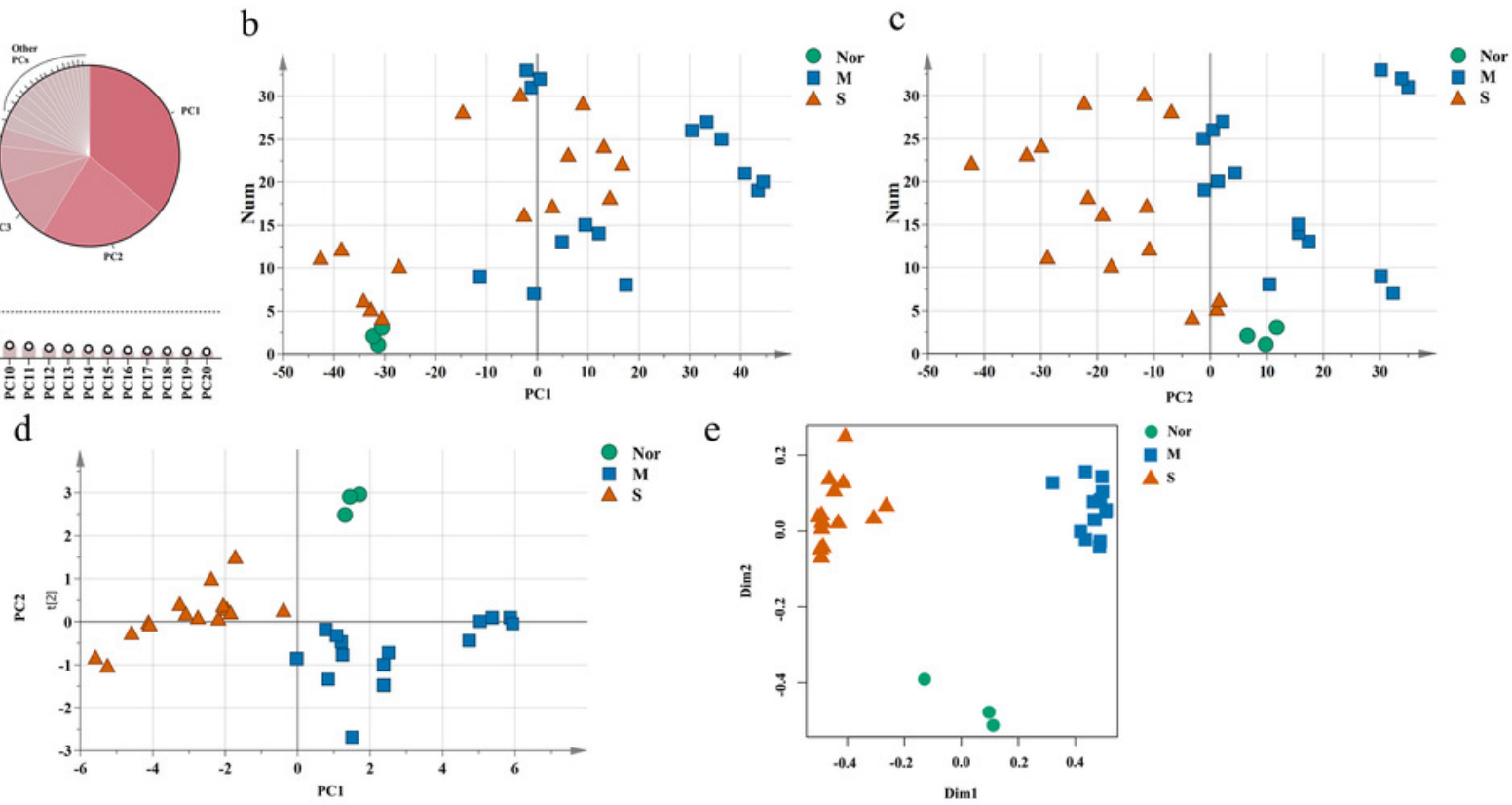

- Nor $\Delta \mathrm{s}$ 


\section{Figure 4}

Fig.4. Time-series analysis of differentially expressed genes in mild contusion groups.

(a) Unsupervised hierarchical clustering of gene expression profiles. (b) Principal component analysis of differentially expressed genes in all biological replicates. (c-e) Top 3 significant genes according to HotellingT2 value; $1 \mathrm{~A}$ (in bluish green), 2B (in blue), and $3 \mathrm{C}$ (in vermilion) represent the three biological replicates at each time point. (f) Heatmap of the expression levels of the top 60 differentially expressed genes in the mild injury groups, showing that the expression can be clustered into four temporal patterns. (g) The performance of t-SNE based on these 60 genes.

a

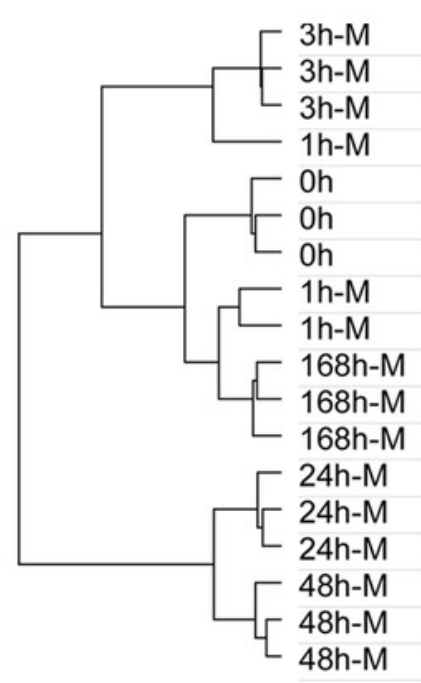

b

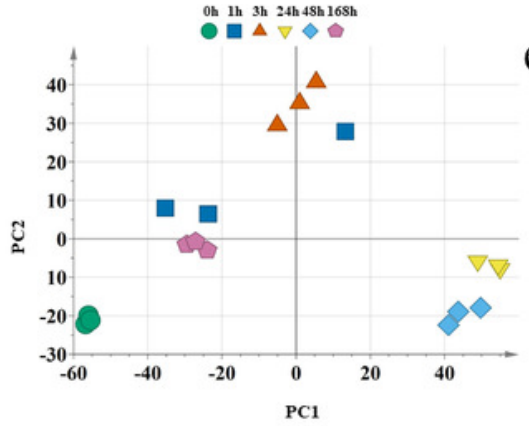

c
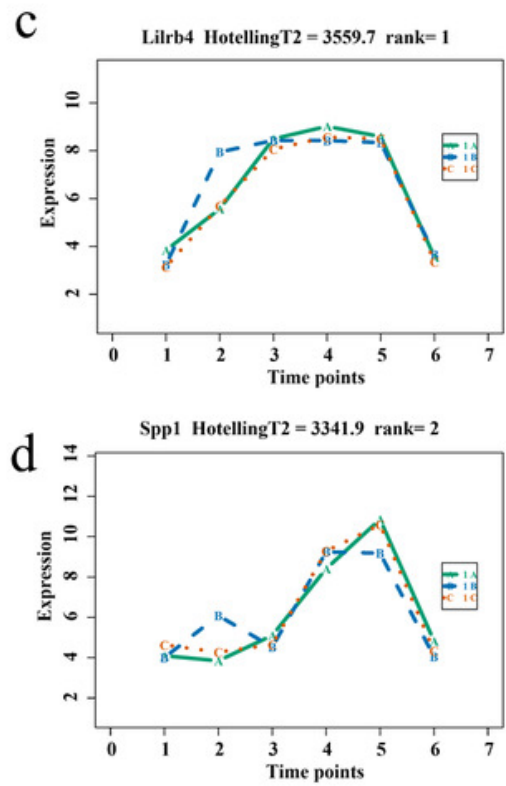

e

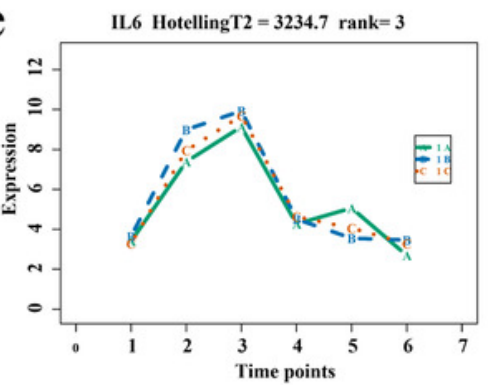

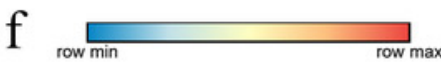

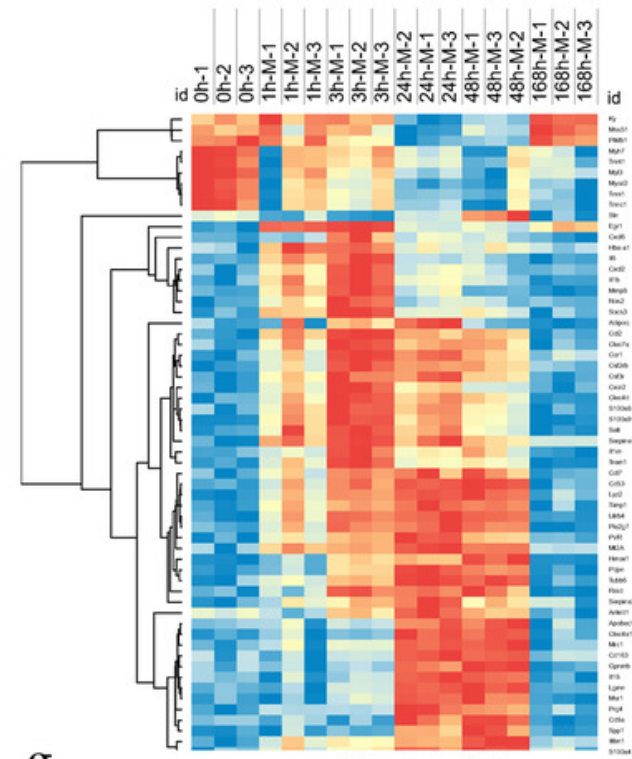

$\mathrm{g}$

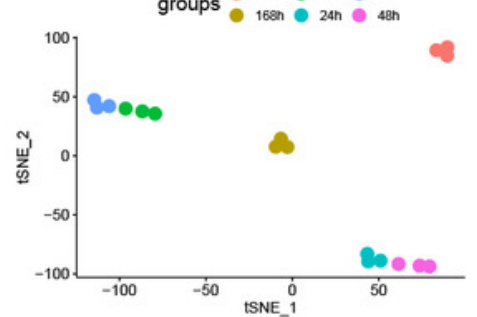


Figure 5

Fig.5. Functional analysis of top 60 differentially expressed genes in mild injury.

(a) Go enrichment; BP, biological progress; CC, cellular component; MF, molecular function.

(b) Kegg analysis using the clueGo plugin in Cytoscape 3.7.2. (c) Functional network of

genes. The more reddish the node, the higher the connectivity degree of the node.

a

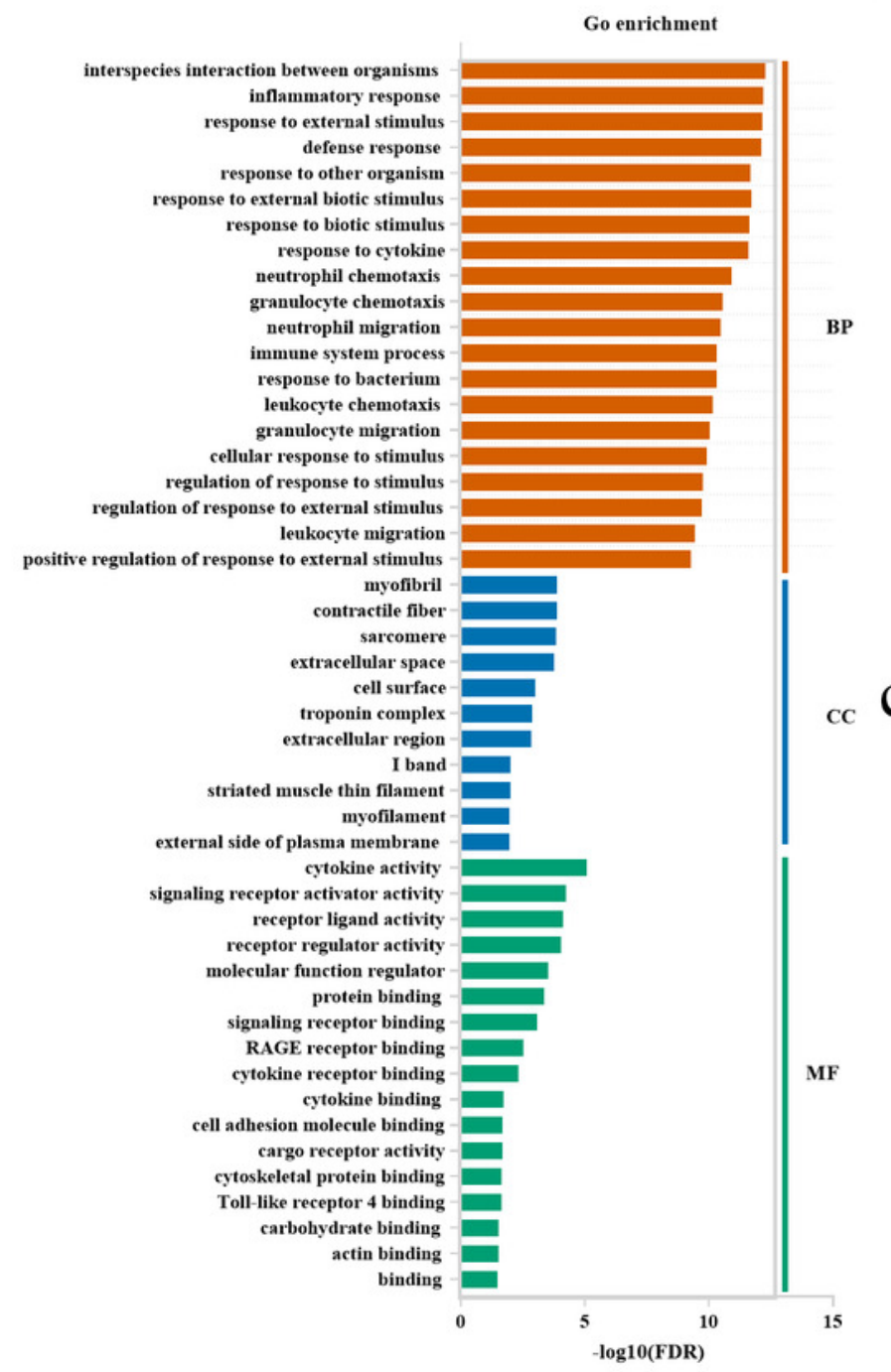

b

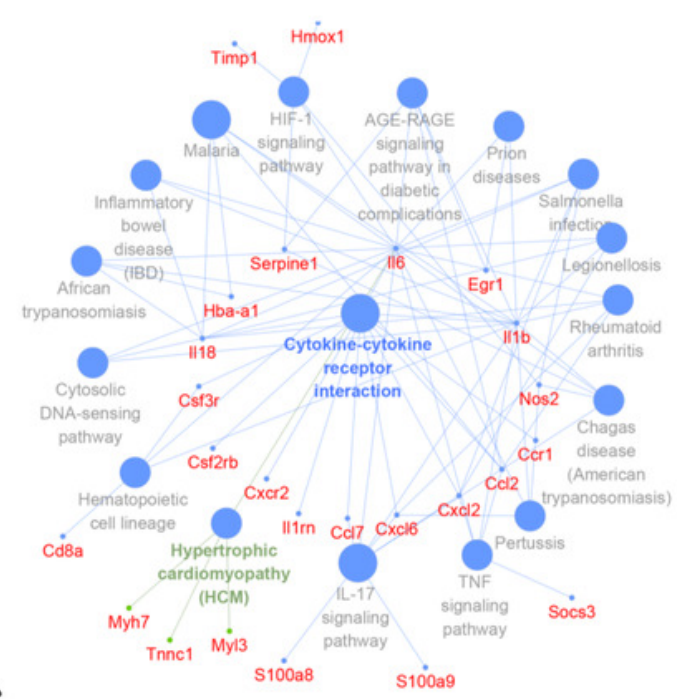

C

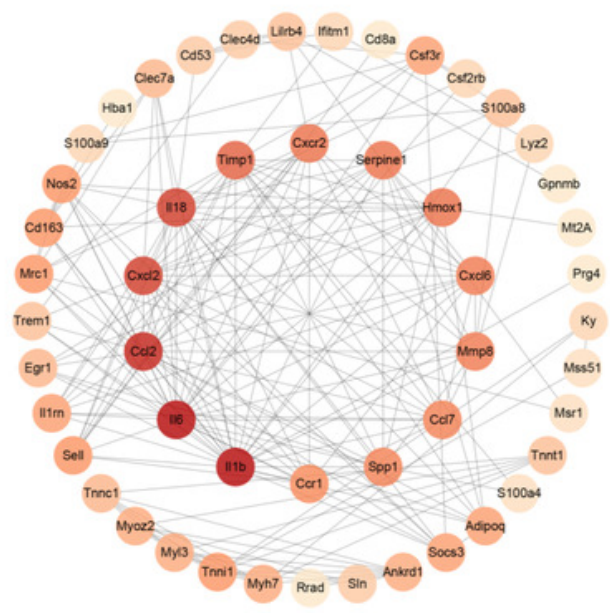


Figure 6

Fig.6. Time-series analysis of differentially expressed genes in severe contusion groups.

(a) Unsupervised hierarchical clustering of gene expression profiles. (b) Principal component analysis of differentially expressed genes in all biological replicates. (c-e) Top 3 significant genes according to HotellingT2 value; $1 \mathrm{~A}$ (in bluish green), 2B (in blue), and $3 \mathrm{C}$ (in vermilion) represent the three biological replicates at each time point. (f) Heatmap of the top 60 differentially expressed genes in the severe injury groups. $(\mathrm{g})$ The t-SNE analysis of these 60 genes in the severe injury group.

a

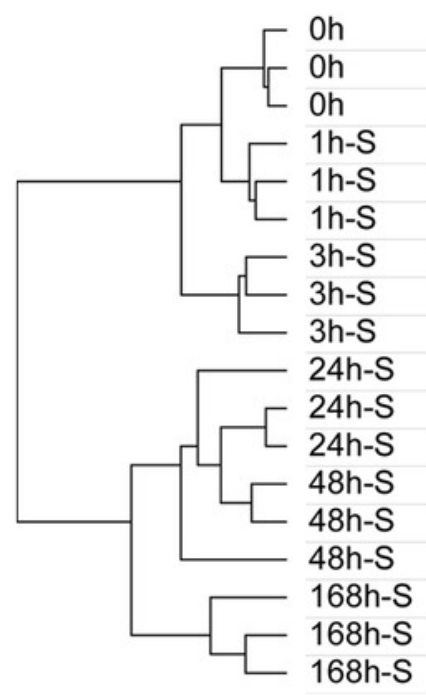

b

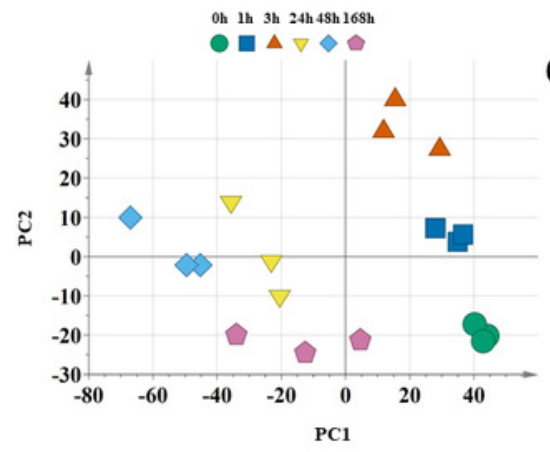

$\mathrm{c}$
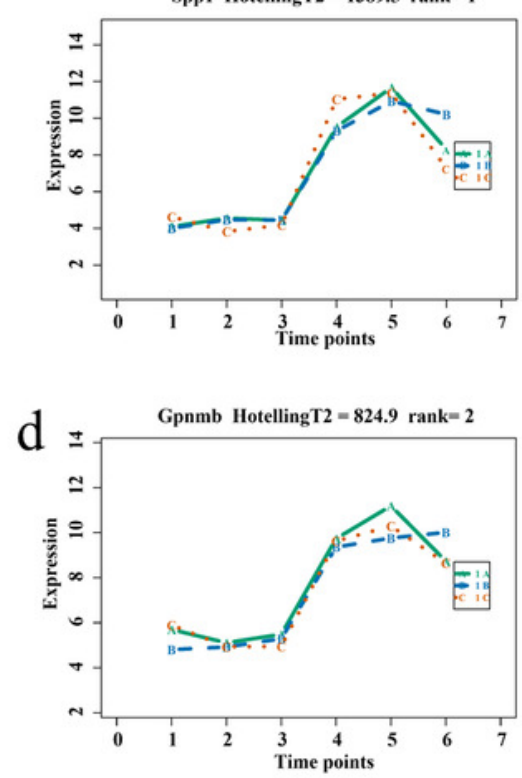

$\mathrm{e}$

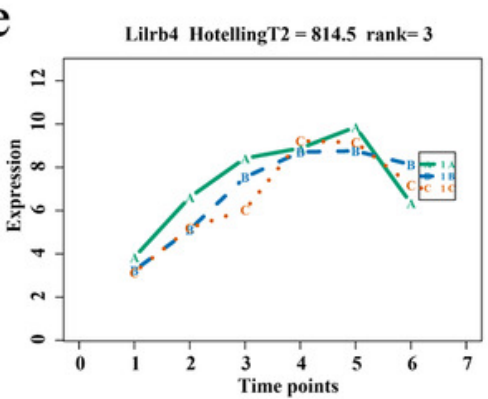

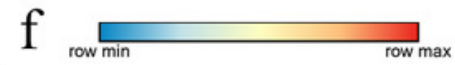

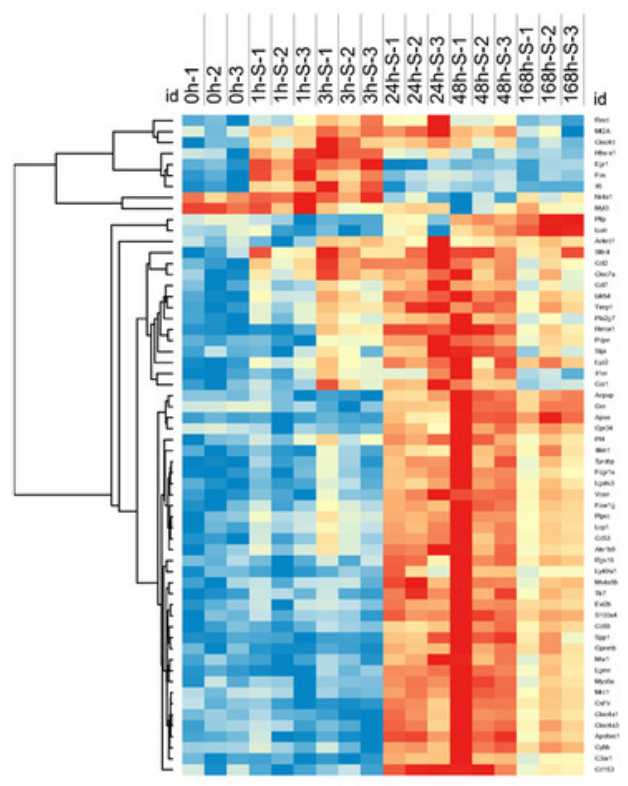

g

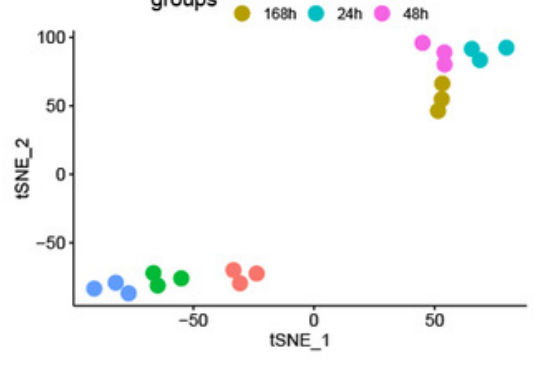


Figure 7

Fig.7. Functional and pathway enrichment of differentially expressed genes in severe injury groups.

(a) Go enrichment; BP, biological progress; CC, cellular component; MF, molecular function.

(b) Kegg analysis by clueGo plugin in Cytoscape 3.7.2. (c) Functional network of these genes. The more reddish the node, the higher the connectivity degree of the node is.

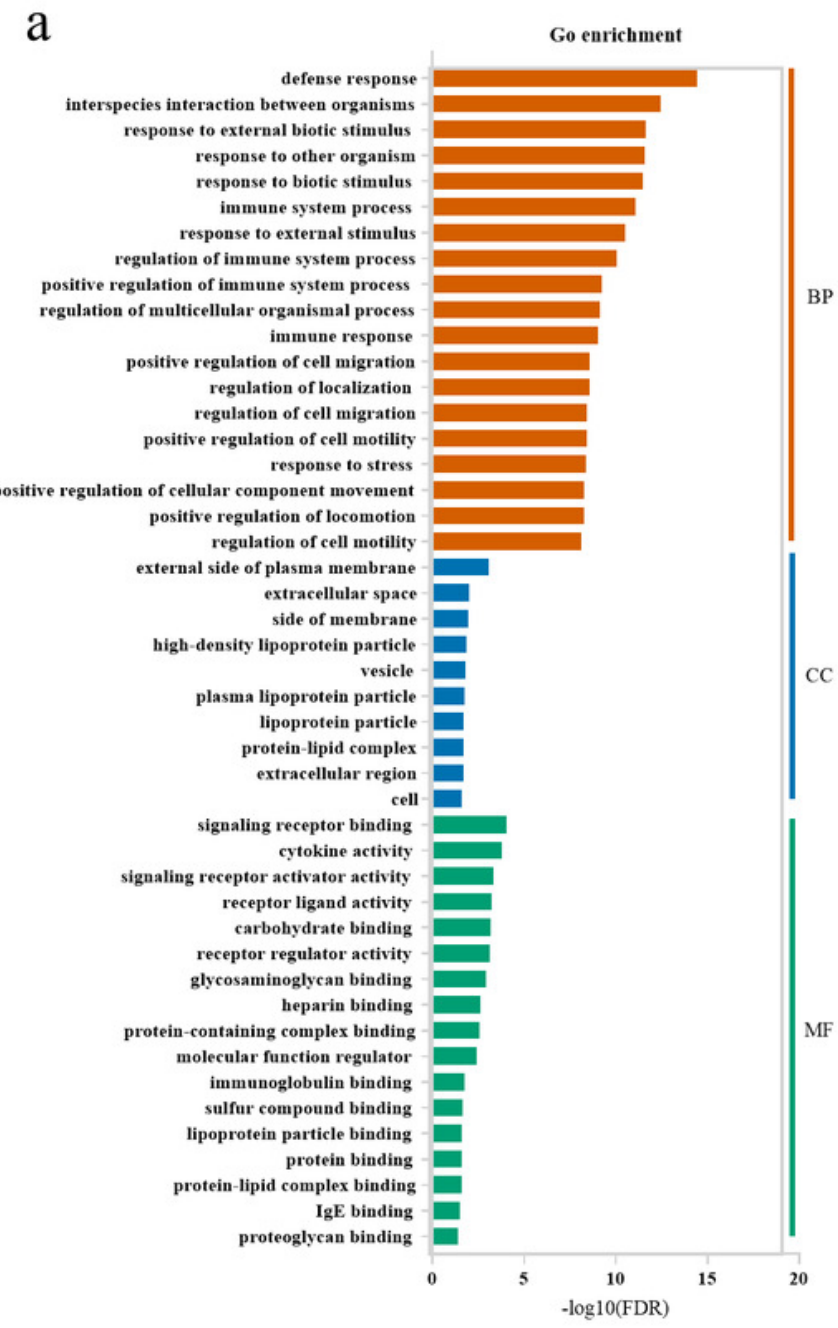

b
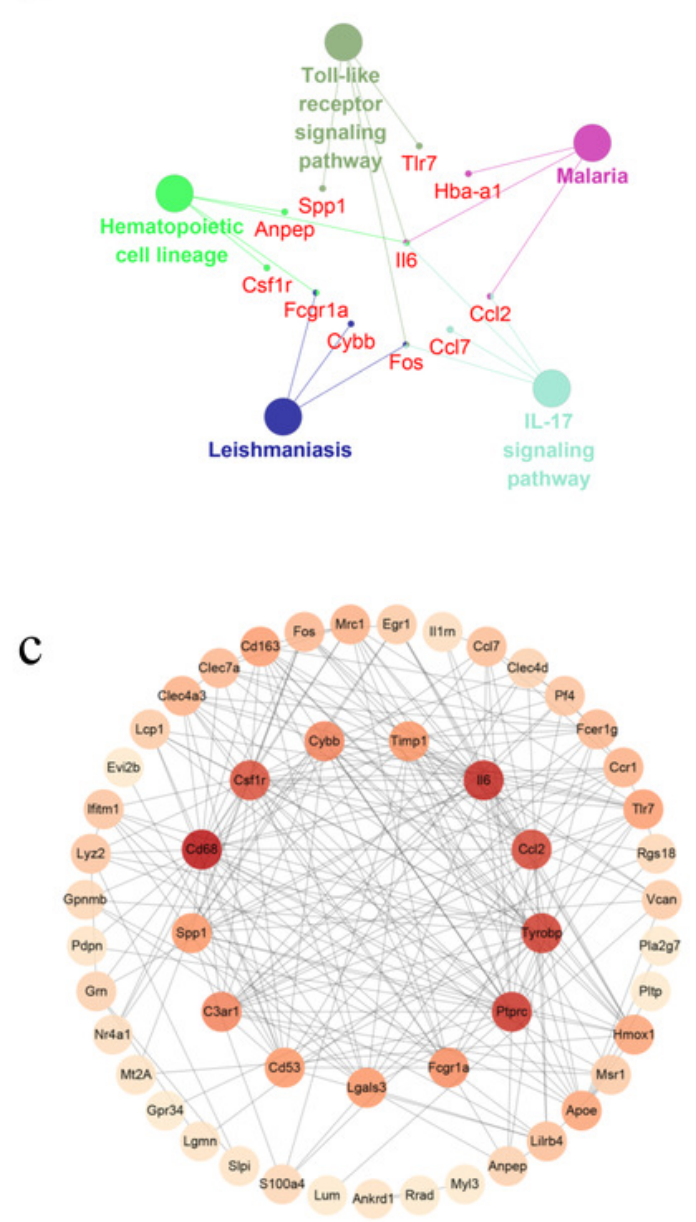
Figure 8

Fig.8. Expression level of Mt2a, Rrad, Gpnmb and Timp1 in microarray analysis (a-d) and RT-qPCR (e-h) in mild group.

a-d $1 A$ (in bluish green), 2B (in blue), and $3 C$ (in vermilion) represent the three biological replicates in microarray analysis .
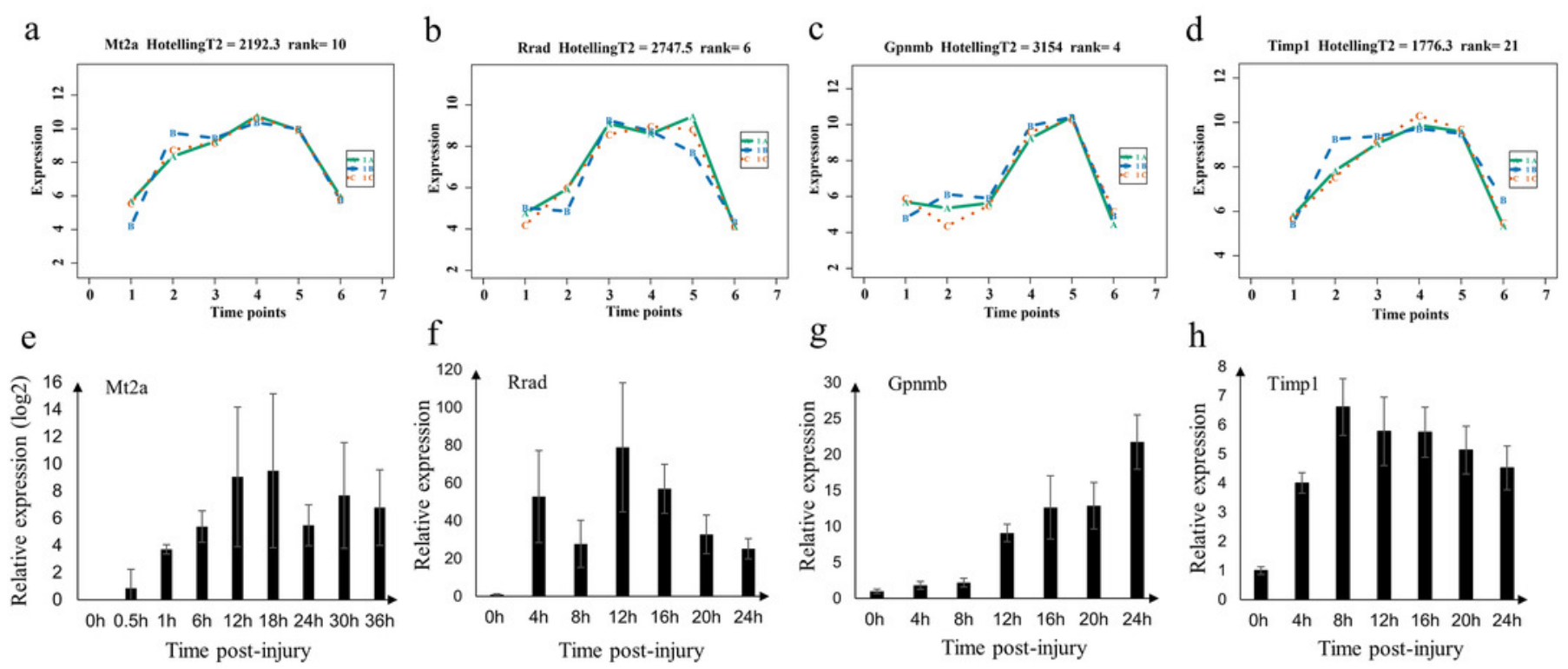
Figure 9

Fig.9. Performance of genes we selected for wound degree and age estimation in Fisher discriminant analysis (FDA) model.

(a) The canonical discrimination functions for the degree of damage. $0 \diamond$ control group, 10 mild injury group, $2 \square$ severe injury group (b, c) The FDA function for wound age estimation in mild and severe injury groups. The Wilks' lambda option was used in the Stepwise method. (0 $\triangleright 0 h, 1 \bigcirc 1 h, 2 \square 3 h, 3 \triangle 24 h, 4 \nabla 48 h, 5 \triangleleft 168 h)$.
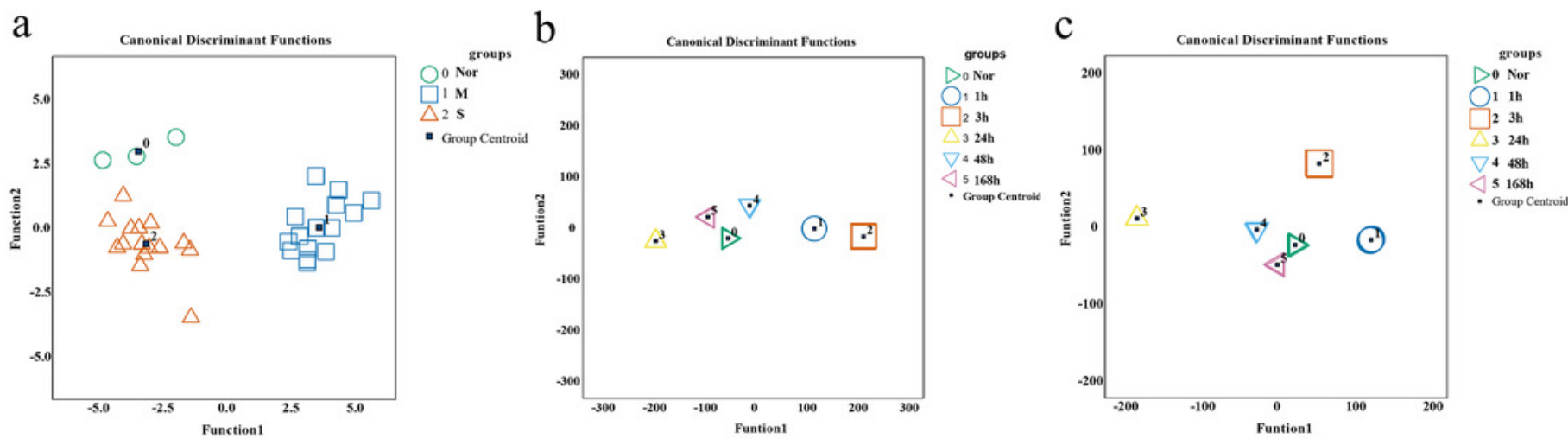